Research Paper

\title{
Selective Killing of Melanoma Cells With Non-Thermal Atmospheric Pressure Plasma and p-FAK Antibody Conjugated Gold Nanoparticles
}

\author{
Byul Bo Ra Choi ${ }^{1}$, Jeong Hae Choi ${ }^{1}$, Jin Woo Hong ${ }^{2}$, Ki Won Song ${ }^{3}$, Hae June Lee ${ }^{4}$, Uk Kyu Kim ${ }^{5 凶}$, Gyoo
} Cheon $\mathrm{Kim}^{1 凶}$

1. Department of Oral Anatomy, School of Dentistry, Pusan National University, Yangsan 626-870, Republic of Korea;

2. Department of Korean Internal Medicine, School of Korean Medicine, Pusan National University, Yangsan 626-870, Republic of Korea;

3. Department of Biochemistry, College of Life Science and Biotechnology, Yonsei University, Seoul 120-749, Republic of Korea;

4. Department of Electrical Engineering, Pusan National University, Busan 609-735, Republic of Korea;

5. Department of Oral \& Maxillofacial Surgery, School of Dentistry, Pusan National University, Yangsan 626-870, Republic of Korea.

$\triangle$ Corresponding authors: Uk Kyu Kim, Department of Oral \& Maxillofacial Surgery, School of Dentistry, Pusan National University, Yangsan 626-870, Republic of Korea. Email: kuksjs@pusan.ac.kr; Tel: 82-55-360-5112; fax: 82-55-360-5104. Gyoo Cheon Kim, Department of Oral Anatomy, School of Dentistry, Pusan National University, Yangsan 626-870, Republic of Korea. Email: ki91000m@pusan.ac.kr; Tel: 82-51-510-8243; fax: 82-51-510-8241.

(C) Ivyspring International Publisher. This is an open access article distributed under the terms of the Creative Commons Attribution (CC BY-NC) license (https://creativecommons.org/licenses/by-nc/4.0/). See http://ivyspring.com/terms for full terms and conditions.

Received: 2017.03.16; Accepted: 2017.07.05; Published: 2017.09.04

\begin{abstract}
Melanomas are fast growing high-mortality tumors, and specific treatments for melanomas are needed. Melanoma cells overexpress focal adhesion kinase (FAK) compared to normal keratinocytes, and we sought to exploit this difference to create a selectively lethal therapy.

We combined gold nanoparticles (GNP) with antibodies targeting phosphorylated FAK (p-FAK). These conjugates (p-FAK-GNP) entered G361 melanoma cells and bound p-FAK. Treatment with p-FAK-GNP decreased the viability of G361 cells in a time dependent manner by inducing apoptosis. To maximize the preferential killing of G361 cells, non-thermal atmospheric pressure plasma was used to stimulate the GNP within p-FAK-GNP. Combined treatment with plasma and p-FAK-GNP showed much higher lethality against $\mathrm{G} 361$ cells than $\mathrm{HaCaT}$ keratinocyte cells. The p-FAK-GNP induced apoptosis over 48 hours in G361 cells, whereas plasma and p-FAK-GNP killed G361 cells immediately.

This study demonstrates that combining plasma with p-FAK-GNP results in selective lethality against human melanoma cells.
\end{abstract}

Key words: Gold nanoparticles, Focal adhesion kinase, Melanoma, Tumor targeting, Non- thermal atmospheric pressure plasma.

\section{Introduction}

Melanoma can occur anywhere melanin-producing cells exist, and thus it is often found on the mucosal surface of the vulva, on the anorectal mucosa, in the nasal cavity, or in the oral cavity. The incidence of melanoma has increased over the last four decades, and in the case of metastatic melanoma, patients have a very poor prognosis with a low five-year survival rate [1-4].

Several techniques have been established for cancer treatment, including chemotherapies, radiotherapies, and immunotherapies. Recently, targeted immunotherapies using antibodies have been the subject of investigation, and these are referred to as next generation therapies [5]. When combined with a radioisotope or other toxin, antibodies against antigens enriched on cancer cells can selectively kill those cells and effectively treat the cancer [6, 7]. However, non-specific reactivity to normal cells can create unwanted toxicity, and the immunotoxins can cause secondary antibody-antigen interactions with existing antibodies, thus limiting their effectiveness against cancer cells. Recently, nanoparticles have been applied in cancer treatment to avoid the problems plaguing chemical anticancer 
drugs. Advances in nano-technology have resulted in the use of gold nanoparticles (GNP) as diagnostic and drug delivery tools $[8,9]$. The GNP is suitable for use as a marker of tumor cells, and if antigen-specific ligands or antibodies are bound to nanoparticles, the effect can be maximized while avoiding the pharmacological side-effects.

Plasma is a highly reactive gas with potential biomedical applications. A number of previous reports have shown plasma to be effective for killing cancer cells, sterilizing instruments, causing coagulation, facilitating wound healing, and rejuvenating skin [10-12]. However, plasma alone is unable to kill cancer cells selectively, since there is no way for it to target cancer cells over normal cells. Plasma consists of electrons and radical ions in an electric field, and because of this it can react with and activate GNP $[13,14]$. In this study, plasma was used along with GNP conjugated to antibodies targeting cancer-cell specific proteins to kill cancer cells selectively [15]. Cancer specific antibody-conjugated GNP preferentially bind to cancer cells that express the target antigen at high levels. The stimulation of the GNP by plasma results in severe stress to the cancer cells, leading to cell death. Therefore, we manufactured a GNP-antibody conjugate using a tumor specific antibody known to be both safe and able to enter cells.

We chose to use an antibody targeting focal adhesion kinase (FAK), a protein that is over-expressed in melanoma cells [16]. FAK is a tyrosine kinase that is involved in early integrin/extracellular matrix (ECM) signaling that has been shown to modulate cancer cell migration, invasion, and survival [17-19]. Increased expression of FAK is crucial for the survival, growth, and metastasis of melanoma cells [20]. We expected that conjugation of an antibody against phosphorylated-FAK (p-FAK) to GNP (p-FAK-GNP) would result in a conjugate with high selectivity for melanoma cells. The plasma-stimulated p-FAK-GNP should inhibit the activity of FAK and thereby inhibit cellular functions within melanoma cells specifically, leading to their death. Therefore, this study was performed to investigate the possibility of selectively killing G361 human skin malignant melanoma cells with p-FAK-GNP and plasma, and to understand the molecular pathways involved.

\section{Materials and Methods}

\section{Materials}

The following chemicals and reagents were obtained from the indicated companies: The RNase A, proteinase $\mathrm{K}$, leupeptin, aprotinin, PI, phenylme- thylsulfonyl fluoride (PMSF), and Ponceau S were purchased from Sigma (St Louis, MO, USA). SuperSignal West Femto enhanced chemiluminescence western blotting detection reagent was from Pierce (Rockford, IL, USA).

\section{Antibodies}

The monoclonal mouse anti-human p-FAK antibody was obtained from Millipore (CA, USA). The rabbit polyclonal anti-human p-paxillin antibody was purchased from Cell Signaling Technology, Inc. (Boston, MA). The rabbit polyclonal anti-human NEU and DFF 45 antibodies were purchased from Santa Cruz Biotechnology (CA, USA). Monoclonal mouse anti-human NEU, FAK, BAX, Bcl-2, GAPDH, Caspase 9, and Caspase 3 antibodies were purchased from Santa Cruz Biotechnology (CA, USA). The horseradish peroxidase conjugated goat anti-mouse and anti-Rabbit IgG were purchased from Santa Cruz Biotechnology (CA, USA).

\section{Cell culture}

The G361 and HaCaT cells were cultured in Roswell Park Memorial Institute 1640 media and Dulbecco's modified Eagle medium supplemented with $25 \quad \mathrm{mM}$ Hepes, $100 \mu \mathrm{g} / \mathrm{mL}$ penicillin/streptomycin, $4 \mathrm{mM}$ L-glutamine, and $10 \%$ fetal bovine serum. Cultures were maintained at $37^{\circ} \mathrm{C}$ in a $5 \% \mathrm{CO} 2$ atmosphere in a humidified incubator.

\section{GNP conjugated with antibodies}

To make GNP-antibody conjugates, an aqueous solution of 11-mercaptoundecanoic acid (MUA) (0.1 $\mathrm{mg} / \mathrm{mL}$ ) is added to a colloidal gold suspension and incubated overnight. MUA-modified GNP is then treated with a mixture of $1 \mathrm{mM}$ N-hydroxysuccinimide (NHS) and $1 \mathrm{mM}$ N-ethyl-N'-(3-dimethylaminopropyl) carbodiimide for $20 \mathrm{~min}$. GNP with exposed surface NHS-termini are then incubated with p-FAK antibodies in phosphate buffered saline (PBS) $(1 \mathrm{mM}, \mathrm{pH} 7.0)$ for at least $8 \mathrm{~h}$.

G361 and HaCaT cells were treated with p-FAK-GNP for 20min and then fixed in $4 \%$ paraformaldehyde. Cells were incubated with goat Alexa 488 anti-mouse secondary antibody for $30 \mathrm{~min}$. Fluorescent images were observed under a Zeiss LSM 700 laser-scanning confocal microscope (Göettingen, Germany).

\section{WST assay}

The viability of cells was evaluated in vitro using a WST-1 assay. Cells were seeded at a density of $1 \times$ $10^{4}$ cells per well in a 96-well plate. Cells were then treated with the p-FAK-GNP conjugates. After a 24, 48 , or $72 \mathrm{~h}$ incubation, $10 \mu \mathrm{L}$ WST- 1 reagent was 
added to each well, and the absorbance at $450 \mathrm{~nm}$ was measured $2 \mathrm{~h}$ later using a microplate reader (Quant, Bio-Tek, Highland Park, USA).

\section{Flow cytometry analysis}

The G361 cells, including the p-FAK-GNP-treated cells, were seeded into $35 \mathrm{~mm}$ diameter dishes at densities of $1 \times 10^{4}$ cells/well and incubated for $24 \mathrm{~h}$. Cells were harvested and washed with cold PBS. The cells were then centrifuged at 1500 RPM for $5 \mathrm{~min}$. The cells were then fixed in cold $70 \%$ ethanol for $24 \mathrm{~h}$. The fixed cells were washed with PBS and centrifuged again at $1500 \mathrm{RPM}$ for $5 \mathrm{~min}$. RNase A was added to a concentration of $100 \mu \mathrm{g} / \mathrm{mL}$ to the cells, which were incubated at $37^{\circ} \mathrm{C}$ for $30 \mathrm{~min}$ and resuspended in PI solution $(10 \mu \mathrm{g} / \mathrm{mL})$. Cells were incubated at $4{ }^{\circ} \mathrm{C}$ for $10 \mathrm{~min}$ and analyzed using a BD FACSCanto II flow cytometer (BD Biosciences, San Jose, CA).

\section{Immunofluorescent staining}

Cells were cyto-centrifuged and fixed for $10 \mathrm{~min}$ in $4 \%$ paraformaldehyde, incubated with AIF, cytochrome $\mathrm{c}$ antibody for $1 \mathrm{~h}$ at $37^{\circ} \mathrm{C}$, washed 3 each for $5 \mathrm{~min}$, and then incubated with goat Alexa 488 and 594 conjugated secondary antibody for $1 \mathrm{~h}$ at $37^{\circ} \mathrm{C}$.

Cells were mounted with mounting solution. Fluorescent images were observed and analyzed under Zeiss LSM 700 laser-scanning confocal microscope (Göettingen, Germany).

\section{Western blot analysis}

For protein analysis, the cells were lysed with a lysis buffer $(10 \mathrm{mM}$ Tris/ $\mathrm{HCl}, \mathrm{pH} 7.2,1 \%$ Triton X-100, $150 \mathrm{mM} \mathrm{NaCl}, 5 \mathrm{mM}$ EDTA, $2 \mathrm{mM}$ PMSF, 2 $\mu \mathrm{g} / \mathrm{mL}$ aprotinin, and $2 \mu \mathrm{g} / \mathrm{mL}$ leupeptin) on ice for 1 $\mathrm{h}$. The lysate was clarified by centrifugation at 14000 $\mathrm{RPM}$ for $20 \mathrm{~min}$ at $4{ }^{\circ} \mathrm{C}$, and the supernatant was obtained. The protein content of the lysate was determined using a Bio-Rad Protein Assay (Bio-Rad laboratories Hercules, CA). The samples ( $25 \mu \mathrm{g}$ of lysate) were then boiled for $95{ }^{\circ} \mathrm{C}$ for $5 \mathrm{~min}$, the protein was resolved using polyacrylamide SDS gels and transferred to a PVDF membrane. After transfer, the membranes were blocked with a blocking reagent (5 \% non-fat milk in TBS-T $(20 \mathrm{mM}$ Tris, $150 \mathrm{mM}$ $\mathrm{NaCl}, 0.1 \%$ Tween 20)) for $1 \mathrm{~h}$. The membranes were incubated for $2 \mathrm{~h}$ with the appropriate antibody. The membranes were treated with ECL western blotting reagents for protein detection.

\section{Non-thermal atmospheric pressure plasma source}

A schematic diagram of the experimental setup is shown in Fig. 4. In this case, the size of the device was modified to $10.24 \mathrm{~cm}^{2}$ to allow a wide treatment area. The other design factors were kept to maintain the plasma characteristics. The mask pattern was etched using a wet etching technique on $\mathrm{Cu}$ electrodes, which were surrounding both sides of a polytetrafluoroethylene (PTFE) dielectric surface. The device was connected directly to a high voltage AC power source $(15 \mathrm{kV}, 22 \mathrm{kHz})$. The front side of the device was grounded for safety reasons. The plasma operated in ambient air with $500 \mathrm{~V}$ (RMS) applied voltage. The applied voltage was controlled and maintained at a specific value to avoid indiscriminate cell death. For the treatment of G361 or HaCaT cells (4 $\times 10^{4}$ cells) with plasma, the cells were seeded on the glass cover slips (12 $\mathrm{mm}$ diameter) as shown in Fig. 4A. Just before plasma treatment, the cover glasses containing the cells were placed under a thin, tetragonal plasma generator (Fig. $4 \mathrm{~b}$ ). The distance between cells and the device was kept at $2 \mathrm{~mm}$ during the $30 \mathrm{~s}$ treatment. For the $24 \mathrm{~h}$ before the treatment, cells were incubated in growth media with or without p-FAK-GNPs. Just before the treatment, the cover glasses were washed with PBS to remove non-selectively bound and unbound p-FAK-GNP conjugates.

\section{Transmission electron microscopy of cell morphology}

G361 cells were grown on Aclar film coated with collagen. After treatments as indicated, the G361 cells were fixed at $4{ }^{\circ} \mathrm{C}$ for $1.5 \mathrm{~h}$ with a fixative of $4 \%$ PFA and $2.5 \%$ glutaraldehyde in $0.1 \mathrm{M}$ phosphate buffer (pH 7.4). Cells were then rinsed with phosphate buffer and after fixation in $1 \%$ osmium tetroxide, the cells were put through a graded ethanol series. Cells were removed from the Aclar film and placed in a glass scintillation vial containing propylene oxide (PO). Dehydration was performed with three sequential incubations, each for $5 \mathrm{~min}$ in fresh $\mathrm{PO}$, and one final incubation in fresh $\mathrm{PO}$ for $10 \mathrm{~min}$. Cells were infiltrated with a mixture of 1:1 Epon:PO for $1 \mathrm{~h}$, then with a 2:1 mixture for $2 \mathrm{~h}$, then with pure Epon for $2 \mathrm{~h}$, and finally with Epon and an added accelerator for 42-48 h. Cells were embedded in Epon, and ultrathin sections were examined using a JEM 1200EX-II transmission electron microscope (Jeol; Tokyo, Japan).

\section{Statistical Analysis}

Three independent experimental replicates were performed for each experiment, and the standard deviation is indicated with error-bars in the Figures. The treated, co-treated, and control group results were compared for statistically significant differences $(\mathrm{p}<0.001,0.01$, and 0.05) using paired T-tests that were performed with the PASW Statistics 18 (formerly SPSS) software. 


\section{Results}

\section{The levels of phosphorylated FAK in G361 melanoma cells}

The levels of p-FAK in G361 human melanoma cells were compared with those in $\mathrm{HaCaT}$ normal human keratinocytes by western blot analysis. G361 cells had large amounts of p-FAK protein, whereas p-FAK was barely detectable in normal $\mathrm{HaCaT}$ cells (Fig. 1A).

An immunofluorescence assay was used to elucidate whether GNP conjugated with Alexa Fluor 488 antibodies could flow into cells. As shown in Fig. 1B, GNP were observed in the cytosol of both G361 and $\mathrm{HaCaT}$ cells after four hours of incubation. When cells were treated with p-FAK-GNP that were similarly fluorescently labeled, the p-FAK-GNP were mainly localized at cell membranes of G361 cells, where the p-FAK likely localized (Fig. 1C). In the case of $\mathrm{HaCaT}$, the localization pattern of the p-FAK-GNP was very similar to that of GNP themselves, consistent with the observed lack of FAK expression.

\section{Preferential inhibition of melanoma cells by p-FAK-GNP}

As shown in Fig 2A, treatment with p-FAK antibodies inhibited the viability of G361 cells in a time-dependent manner ( 24 h, 94 \% viability; 48 h, 57 $\%$ viability; and $72 \mathrm{~h}, 11 \%$ viability). Interestingly, treatment of G361 cells with an equal concentration of p-FAK-GNP led to more rapid growth inhibition (24 h, $72 \%$ viability; 48 h, $14 \%$ viability; and 72 h, $2 \%$ viability; Fig. 2B). In contrast, the viability of HaCaT cells was barely affected by p-FAK-GNP (Fig. 2C). Therefore, the p-FAK-GNP showed anti-proliferative effects against G361 cells specifically.

The changes in cellular signaling proteins known to be activated by FAK were examined in G361 cells treated with p-FAK-GNP by western blot analysis. The level of activated paxillin, the main target protein of FAK, was significantly decreased following treatment with p-FAK-GNP, although the levels of FAK and p-FAK were also slightly decreased. Furthermore, levels of NEU, a protein upstream of FAK, and phosphorylated-NEU (p-NEU) were significantly decreased at $24 \mathrm{~h}$, and then undetectable at $48 \mathrm{~h}$ in G361 cells treated with p-FAK-GNP. This result suggests that the p-FAK-GNP can block other signaling pathways (Fig. 2D)

\section{Induction of apoptosis in $\mathbf{G} 361$ cells by p-FAK-GNP}

G361 cells were treated with p-FAK-GNPs for 0 to $48 \mathrm{~h}$ and their cell cycle progress was monitored using flow cytometry with propidium iodide (PI) staining (Fig. 3A). There were no significant changes in the cell cycle until apoptosis began to increase. A significant increase was detected in the sub-G1 population of cells exposed to p-FAK-GNP for more than $24 \mathrm{~h}$. Interestingly, the number of cells in all cell cycle phases (G1, S, and G2/M) gradually decreased as the number of apoptotic cells increased.

After p-FAK-GNP treatment, the subcellular localizations of mitochondrial apoptosis-related factors were examined to confirm apoptosis at the molecular level. Immunofluorescence microscopy showed that cytochrome c (Fig. 3B) and AIF (Fig. 3C) in the control cells were localized in punctuate patterns, whereas those proteins were diffusely distributed in p-FAK-GNP-treated cells indicating their release from the mitochondria into the cytosol. In particular, the distribution of AIF was primarily cytoplasmic in the control cells, but AIF was observed in the nuclei of cells treated with p-FAK-GNP.

The changes of the expression of proteins related with apoptosis, such as a BAX, Bcl-2, caspase-3, caspase-9, PARP-1 and DFF 45 (also known as ICAD) were examined by western blot assay. As shown in Fig. 3D, sequential activation of apoptotic proteins (caspase-9, caspase-3, PARP-1, and DFF 45) was observed after p-FAK-GNP treatment in G361 cells. A significant decrease in the level of the anti-apoptotic protein Bcl-2, coupled with an increase in the apoptosis-promoting BAX, was detected in cells treated with p-FAK-GNP for $48 \mathrm{~h}$.

\section{The selective killing effect of plasma and p-FAK-GNP on G361 cells}

Next, we examined the effect of plasma on p-FAK-GNP-mediated cell death. As shown in Fig. $5 \mathrm{~A}$, the cells exposed to plasma showed mild induction of apoptosis (about $19.5 \%$ of G361 cells, and $14 \%$ of HaCaT cells). When G361 cells treated with p-FAK-GNP were exposed to the plasma, the death rate was significantly increased to $72.6 \%$. In contrast, in the case of HaCaT cells, the combined treatment of p-FAK-GNP and plasma resulted in only a moderate increase in death rate $(23.8 \%)$.

Transmission electron microscopy was performed in order to explore the effect of plasma with or without p-FAK-GNP on the morphology of G361 cells. As shown in Fig. 5B, no significant morphological changes were detected in the cells treated with plasma alone. However, cells treated with a combination of plasma and p-FAK-GNP formed many vacuoles and had disrupted cellular membranes. 
A

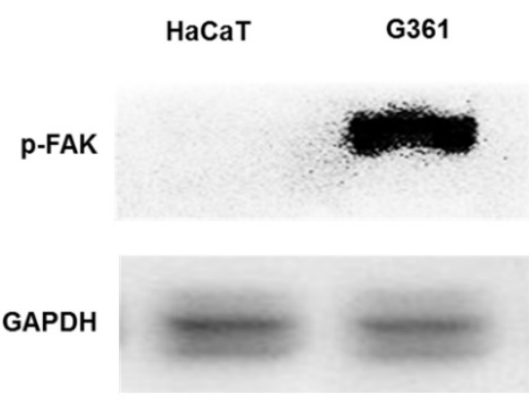

B

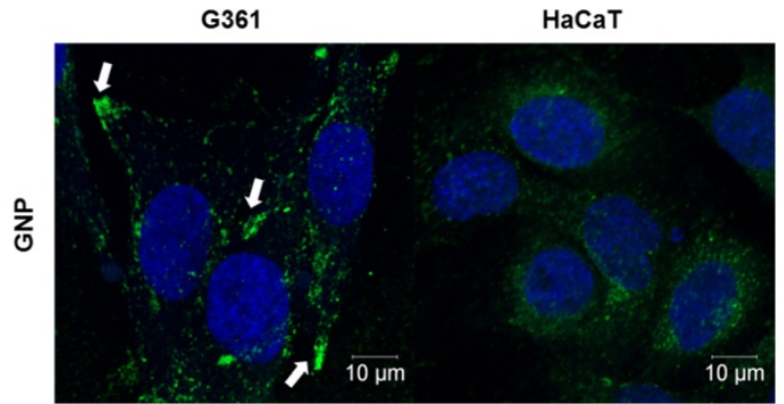

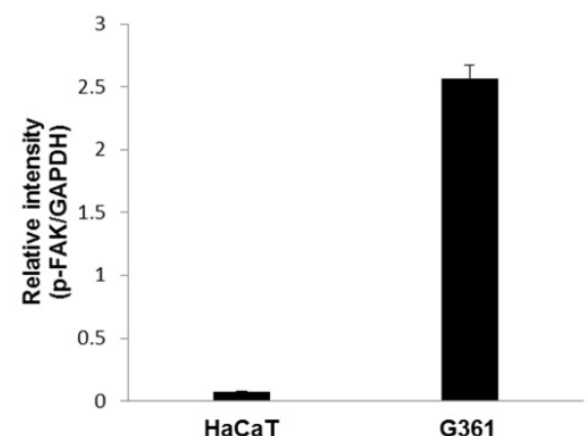

C
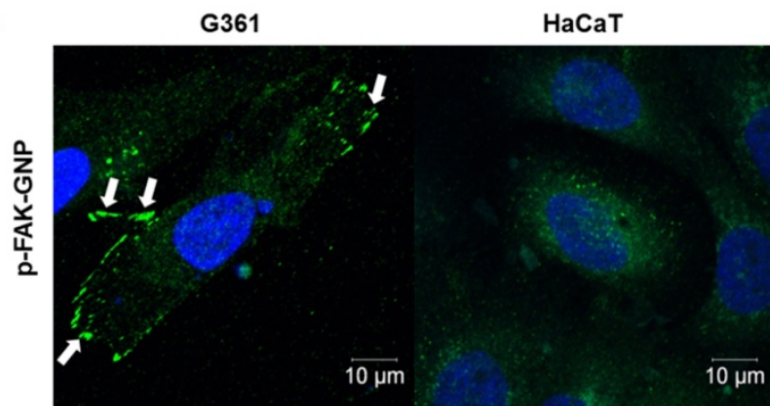

Figure 1. Cellular expression of p-FAK, and uptake of GNP and p-FAK-GNP. (A) Western blot shows the expression of $p-F A K$ protein on HaCaT and G361 ( $p$ $<0.001$ ). (B) The uptake of GNP is shown. The green spots represent GNP absorbed by G361 cells. (C) The uptake of $p-F A K-G N P$ is shown. The $P-F A K-G N P$ specifically bind to P-FAK and generate fluorescence around the G361 cell membranes.

A

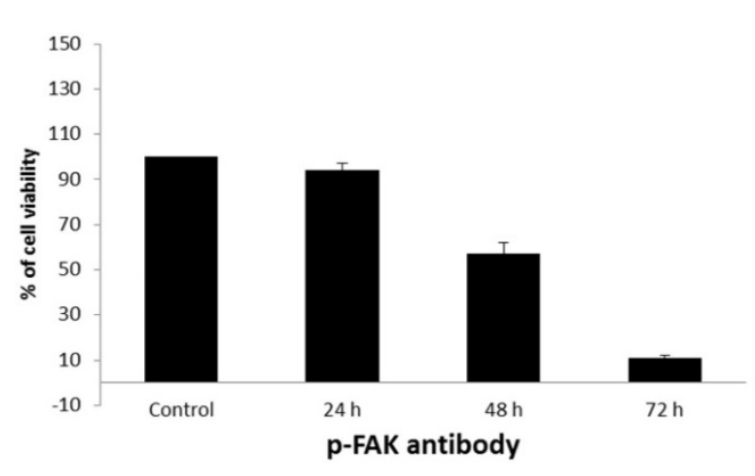

C

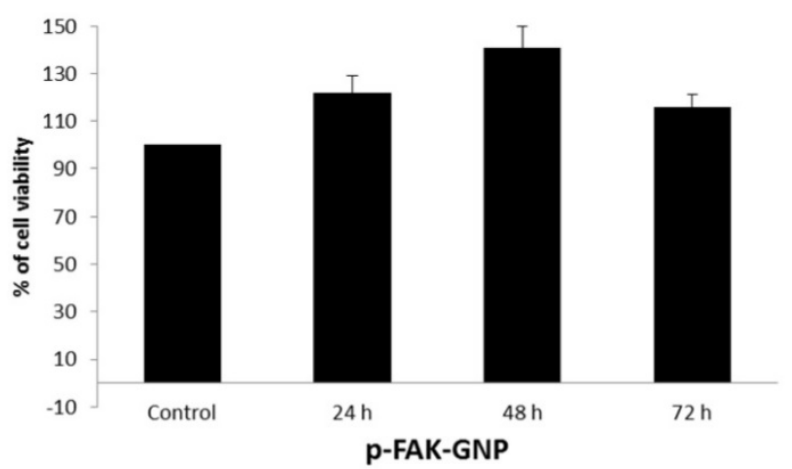

B

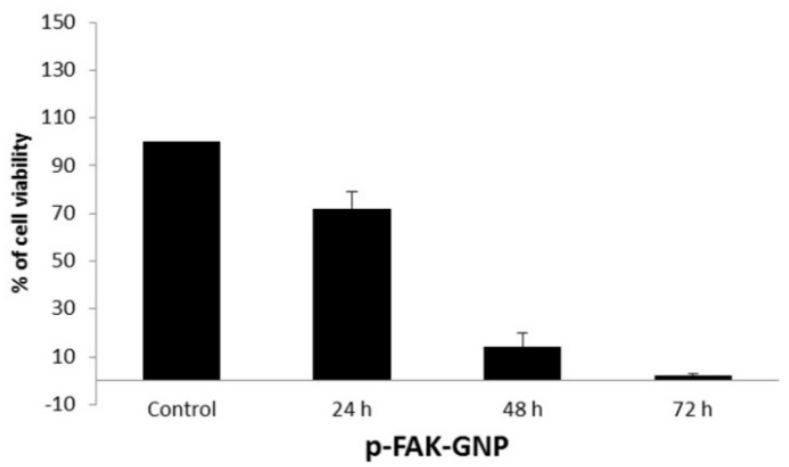

D

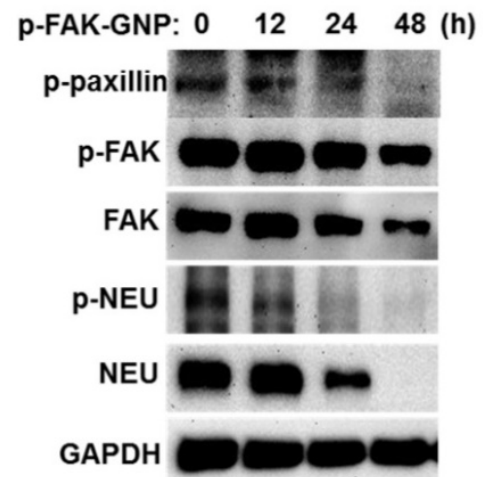

Figure 2. Effects of anti p-FAK and p-FAK-GNP on the proliferation of G361 and HaCaT cells. (A and B) After $24 \mathrm{~h}$ incubation, cell viability was measured using a WST-1 assay. The G361 cells were treated with anti-p-FAK antibodies and p-FAK-GNP for the indicated time periods, and the viability was compared to untreated cells (p-FAK-GNP $24 \mathrm{~h}, p<0.05$; p-FAK-GNP $48 \mathrm{~h}$ and $72 \mathrm{~h}, \mathrm{p}<0.01$; anti-p-FAK $48 \mathrm{~h}$ and $72 \mathrm{~h}, \mathrm{p}<0.05$ ). (C) After $24 \mathrm{~h}$ incubation, the cell viability was measured using a WST-1 assay. The HaCaT cells were treated with P-FAK-GNP for the indicated time periods. (D) After incubating the G361 cells with P-FAK-GNP for the indicated times, the cells were harvested and lysates were analyzed by western blot using p-paxillin, p-FAK, p-NEU, FAK, and NEU antibodies as indicated. 

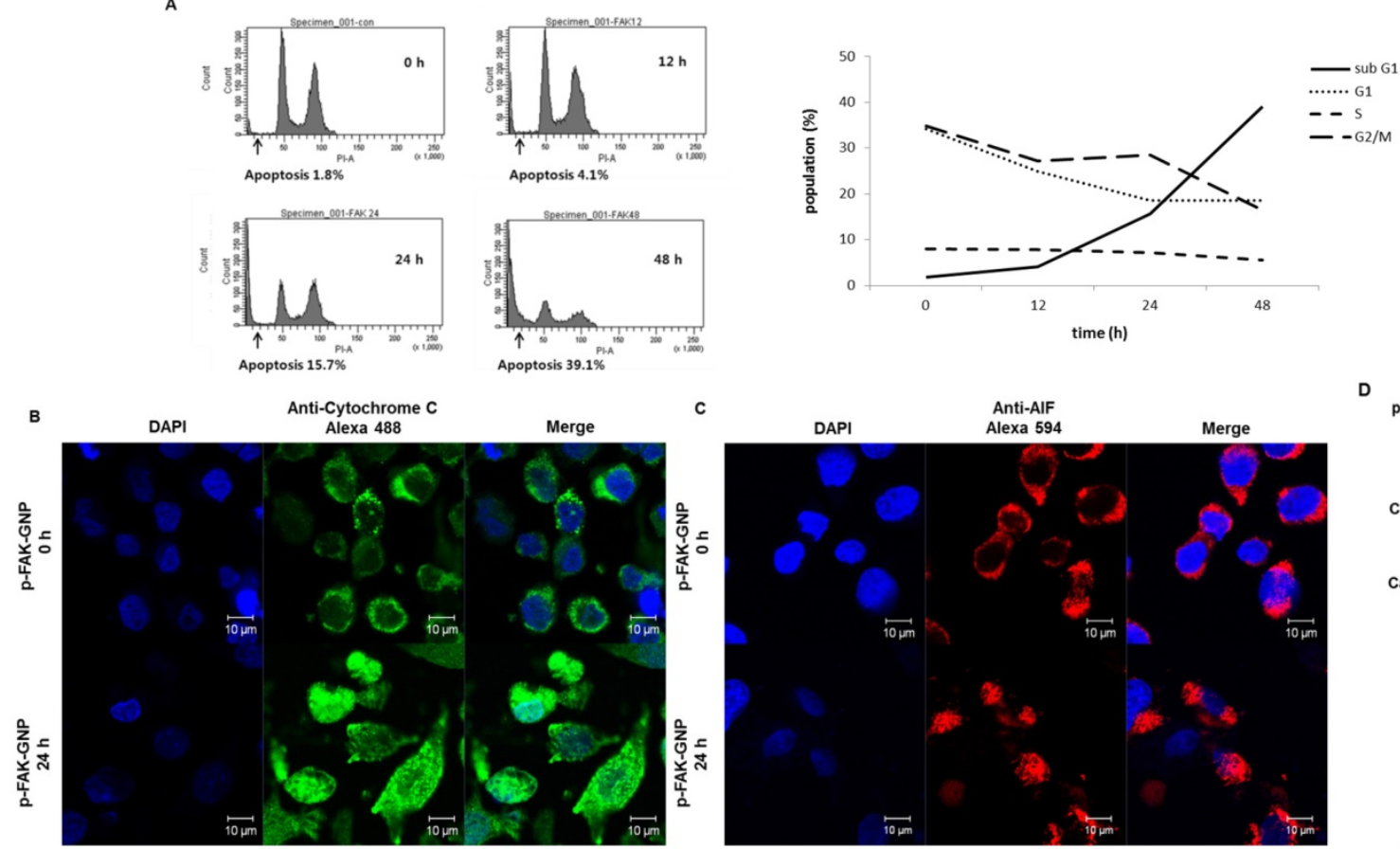

D

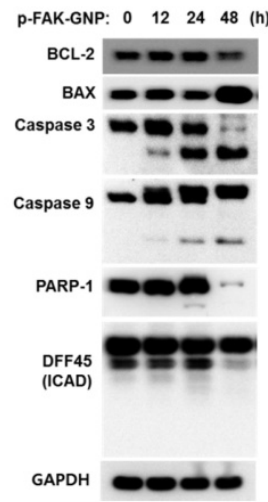

Figure 3. Induction of apoptosis in G361 cells by p-FAK-GNP. (A) The G361 cells were treated with p-FAK-GNP for 12 to $48 \mathrm{~h}$. At each time point, the cells were subjected to FACS analysis using PI solution as indicated. (B) After a $24 \mathrm{~h}$ incubation in the presence (lower panel) or absence (upper panel) of p-FAK-GNP, the translocation of cytochrome $\mathrm{c}$ was visualized by immunocytochemistry using an anti-cytochrome $\mathrm{c}$ antibody. (C) After $24 \mathrm{~h}$ incubation in the presence (lower panel) or absence (upper panel) of p-FAK-GNPs, the subcellular localization of AIF was visualized by immunocytochemistry using an anti-AlF antibody. (D) The G361 cells were incubated in the presence of P-FAK-GNP for the indicated times. The total protein lysates were used for SDS-PAGE followed by western blots using antibodies against BAX, Bdl-2, Caspase 3, Caspase 9, PARP-1, DFF 45, and GAPDH (as a loading control).

A

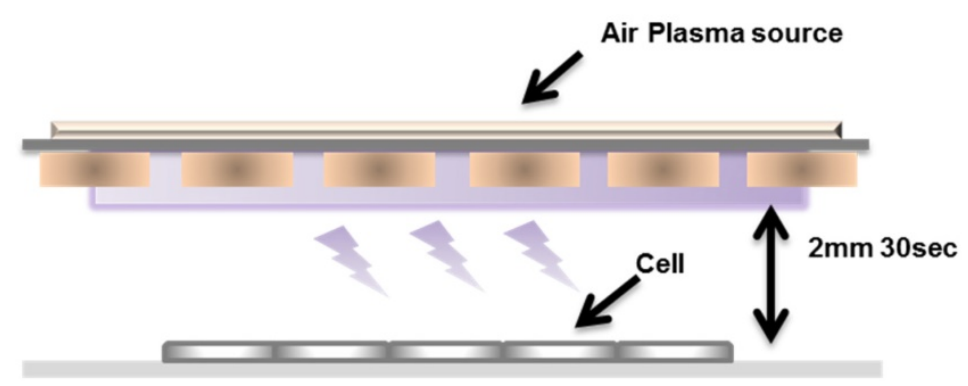

B

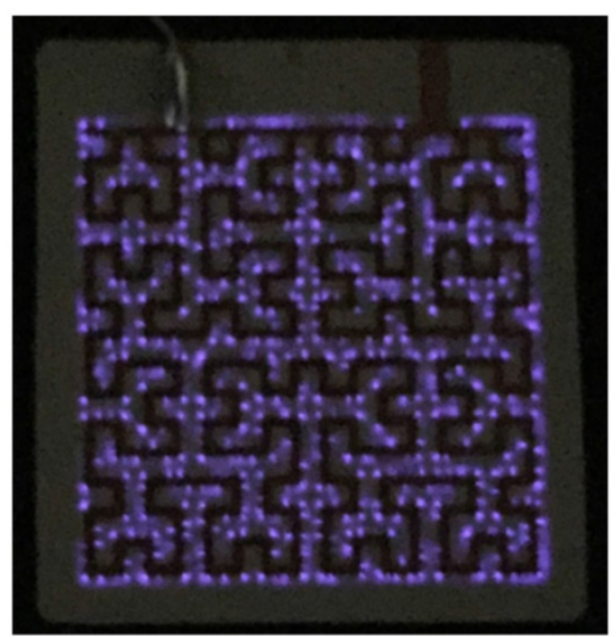

Figure 4. Plasma device characteristics. (A) Schematic diagram of the plasma device and experimental setup. The cells were cultured on glass cover slips for plasma treatment. (B) The air plasma source is shown. 


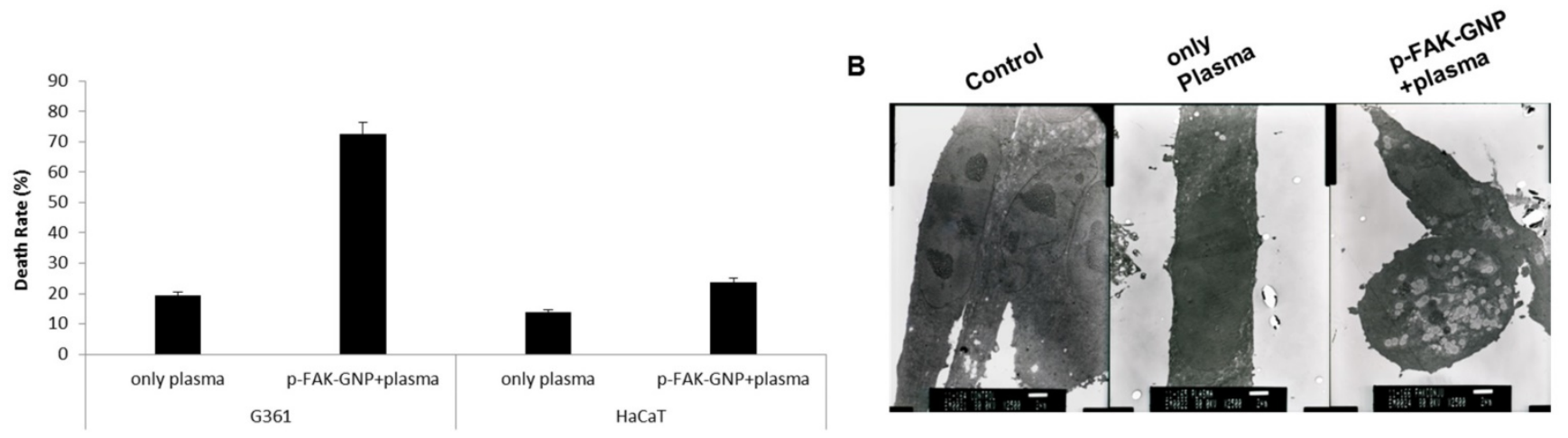

C

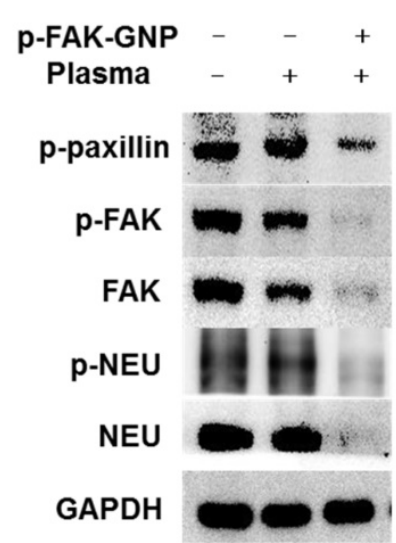

Figure 5. The impact of inducing of p-FAK-GNP with plasma on selective and immediate cell death in G361 cells. (A) After plasma treatment, the cell death rate was measured using Trypan blue assay and compared to the untreated controls (G361: p-FAK-GNP and plasma, $p<0.01$ ). (B) In untreated G361 cells (left panel), cells treated with only plasma (middle panel), and cells treated with p-FAK-GNP and plasma (right panel) $(2500 \times)$. (C) After incubation, the G361 cells were subjected analysis by western blot using NEU, p-NEU, FAK, p-FAK, and p-paxillin antibodies as indicated.

As a final step in this study, the levels of FAK and FAK-regulated proteins were monitored to investigate the molecular mechanisms causing death in G361 cells following treatment with p-FAK-GNP and plasma. In G361 cells without p-FAK-GNP, although plasma treatment slightly decreased the levels of FAK, it did not affect the levels of p-FAK, p-paxillin, NEU, or p-NEU. Conversely, in G361 cells pre-treated with p-FAK-GNP, plasma treatment significantly decreased the levels of FAK, p-FAK, p-paxillin, NEU, and p-NEU (Fig. 5C).

\section{Discussion}

In this study, we investigated the mechanism of death in G361 cells treated with p-FAK-GNP, and cells treated with p-FAK-GNP and plasma treatment. We observed elevated levels of p-FAK in G361 cells compared to HaCaT cells, and confirmed that GNP and p-FAK-GNP can enter cells. The viability and proliferation of G361 cells treated with p-FAK-GNP decreased distinctly over time, whereas the viability of HaCaT cells was barely affected. This suggests that p-FAK is important for G361 melanoma cell survival, and the binding of an anti-p-FAK antibody or p-FAK-GNP inhibits its function. When treated with antibodies against p-FAK alone, the viability of G361 cells decreased after 48 hours. However, in the case of treatment with p-FAK-GNP, the viability of the cells was substantially decreased after 24 hours. This suggests that p-FAK-GNP bind to p-FAK proteins specifically, and the GNP and antibody portions of the p-FAK-GNP may both structurally inhibit the activity of p-FAK, thereby acting synergistically to cause death in G361 cells.

In order to investigate the preferential killing of G361 cells by p-FAK-GNP, we monitored changes in the FAK protein. FAK plays an important role in the construction of focal adhesions between integrin proteins and the ECM. Focal adhesions interact with the cytoskeleton, and are accordingly important for cell morphology, migration, adhesion, and spreading [20]. After several tyrosine residues in FAK have been phosphorylated through the activation of integrin, FAK can then phosphorylate paxillin. Furthermore, paxillin expression has been associated with the expression of NEU in breast cancer cells [22]. NEU is expressed in various cancers including melanoma, and thus it is clinically significant for treatment. When NEU binds a ligand, it activates cell signaling through FAK that promotes the proliferation and metastasis of 
cancer cells [23]. If focal adhesions are destroyed, cells become detached from the ECM and ultimately die. In this study, we observed not only the degradation of FAK and p-FAK, but also the subsequent destruction of p-paxillin, NEU, and p-NEU in G361 cells treated with p-FAK-GNP. These results indicate that targeting FAK with p-FAK-GNPs leads to the degradation of functionally linked proteins.

Analysis of G361 cells treated with p-FAK-GNP using flow cytometry showed that these cells had an increase in the population of sub-G1 hypodiploid cells, indicating that p-FAK-GNP were causing DNA fragmentation. As a result of this, p-FAK-GNP strongly inhibited G361 cell growth by inducing apoptosis. Apoptosis plays an important role in controlling the homeostasis of an organism, and removing cancer cells. Typically, apoptosis involves various molecules such as caspases, death receptors, and Bcl-2 family proteins. It is induced either by death receptors activating the extrinsic pathway, or by mitochondrial damage activating the intrinsic pathway. Bcl-2 family proteins play an important role in controlling the intrinsic pathway, wherein Bcl-2 and Bcl-XL normally suppress apoptosis by inhibiting the release of cytochrome c. Conversely, BAD, BAX, and BAK-1 promote apoptosis by inducing cytochrome c release [24, 25]. These two sets of proteins usually maintain a constant balance within a healthy cell, allowing survival. After treatment with p-FAK-GNP, AIF was released from the mitochondrial membranes into the cytoplasm, and subsequently entered the nuclei of G361 cells. Cytochrome $\mathrm{c}$ also moved from the mitochondrial membrane to the cytoplasm following p-FAK-GNP treatment. Bcl-2 levels were reduced, whereas BAX levels were increased in a time dependent manner. Levels of the active form of caspase 3 increased, and its substrate proteins (PARP-1 and DFF 45) were cleaved. Therefore, the apoptosis of G361 cells induced by p-FAK-GNP was likely a result of the destruction of focal adhesion proteins causing mitochondrial damage and subsequent activation of caspase 3 .

In this study, we demonstrated that p-FAK-GNP preferentially induced apoptosis in G361 cells without affecting normal cells. Although p-FAK-GNP alone were effective against G361 cells, we used plasma in order to maximize the lethality of p-FAK-GNP. Because plasma can react with GNP, it can stimulate p-FAK-GNP that preferentially bind to p-FAK in G361 cells. Thus, stimulated GNP could inhibit the activities of the FAK protein, and consequently p-FAK-GNP lead to the cell death. We investigated the lethal effect of plasma and p-FAK-GNP on G361 cells compared to HaCaT cells. The death rate in G361 cells treated with
p-FAK-GNP and plasma was over three times the death rate of G361 cells treated with only plasma, or $\mathrm{HaCaT}$ cells treated with p-FAK-GNP and plasma. Because HaCaT cells express lower levels of FAK protein, they were also affected by p-FAK-GNP and plasma, but much less than the G361 cells. Considering that cell death was induced by 30 seconds of plasma treatment, the cell death induced by p-FAK-GNP and plasma was induced instantly and selectively. Therefore, this rapid cell death might not be due to apoptosis. This phenomenon is different from apoptosis, and further analysis will be needed to understand it.

In this study, a treatment with a combination of p-FAK-GNP and plasma degraded FAK, p-FAK, p-paxillin, p-NEU, and NEU. While the mere treatment of G361 cells with p-FAK-GNP or plasma did not prevent the expression of FAK, the combined treatment lead to a strong decrease in FAK levels. This result is thought to be due to the energy from the plasma being transferred to the GNP, and the stimulated GNP modifying the structure of the p-FAK protein leading to its inactivation. The destruction of p-FAK reduced the levels of other associated signaling molecules. This momentary loss of cell signaling led to the death of the G361 cells.

\section{Conclusions}

Overall, after applying p-FAK-GNP to cancer cells and normal cells, our results show that treatment with plasma kills G361 melanoma cells effectively and selectively. In treated G361 cells, cell signaling molecules such as FAK, p-paxillin, and NEU were destroyed along with FAK. Therefore, based on the selective killing of G361 cells using p-FAK-GNP and short periods of plasma treatment, the results of this study suggest that our approach could be very effective for treating melanoma. As the interaction between GNP and plasma is still under investigation, it is difficult to demonstrate the exact mechanisms, for which a further research will be done to confirm.

\section{Abbreviations}

FAK: focal adhesion kinase; GNP: gold nanoparticles; p-FAK: phosphorylated FAK; p-FAK-GNP: phosphorylated FAK combined gold nanoparticles; ECM: early integrin/extracellular matrix; MUA: 11-mercaptoundecanoic acid; NHS: N-hydroxysuccinimide; PTFE: polytetrafluoroethylene; EthD-1: ethidium homodimer-1.

\section{Acknowledgment}

This research was supported by the Basic Science Research Program through the National Research Foundation of Korea (NRF) funded by the Ministry of 


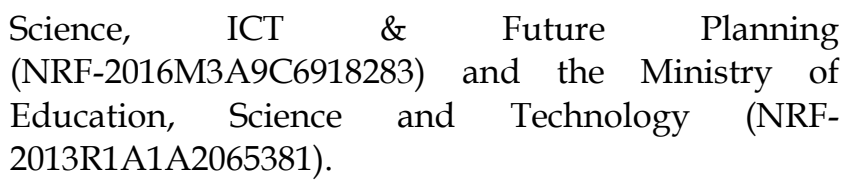

\section{Competing Interests}

The authors have declared that no competing interest exists.

\section{References}

1. Dreiling L, Hoffman S, Robinson WA. Melanoma: epidemiology, pathogenesis and new modes of treatment. Adv Intern Med 1996;41:553-604.

2. Eberle J, Kurbanov BM, Hossini AM, et al. Overcoming apoptosis deficiency of melanoma-hope for new therapeutic approaches. Drug Resist Updat 2007;10:218-34.

3. Hashemi Pour MS. Malignant melanoma of the oral cavity: a review of literature. Indian J Dent Res 2008;19:47-51.

4. Strömberg S, Agnarsd MT, Magnusson K, et al. Selective expression of Syntaxin-7 protein in benign melanocytes and malignant melanoma. J Proteome Res 2009;8:1639-46.

5. Monzon JG, Dancey J. Targeted agents for the treatment of metastatic melanoma. Onco Targets Ther 2012;5:31-46.

6. Schmidt M, McWatters A, White RA, et al. Synergistic interaction between an anti-p185HER-2 pseudomonas exotoxin fusion protein [scFv(FRP5)-ETA] and ionizing radiation for inhibiting growth of ovarian cancer cells that overexpress HER-2. Gynecol Oncol 2001;80:145-55.

7. Risberg K, Fodstad O, Andersson Y. The melanoma specific 9.2.27 PE immunotoxin efficiently kills melanoma cells in vitro. Int $\mathrm{J}$ Cancer 2009;125:23-33.

8. Service RF. Materials and biology. Nanotechnology takes aim at cancer. Science 2005;310:1132-4.

9. Rayavarapu RG, Petersen W, Ungureanu C, et al. Synthesis and bioconjugation of gold nanoparticles as potential molecular probes for light-based imaging techniques. Int J Biomed Imaging 2007;2007:29817.

10. Choi JH, Lee HW, Lee JK, et al. Low temperature atmospheric plasma increases the expression of anti-aging genes of skin cells without causing cellular damages. Arch. Dermatol Res. 2013;305: 133-40.

11. Fridman G, Shereshevsky A, Jost MM, et al. Floating Electrode Dielectric Barrier Discharge Plasma in Air Promoting Apoptotic Behavior in Melanoma Skin Cancer Cell Lines. Plasma Chem Plasma Process 2007;27:163-76.

12. Fridman $G^{1}$, Fridman $G^{2}$, Gutsol A, et al. Applied Plasma Medicine. Plasma Process Polym 2008;5:503-33

13. Kong MG, Keidar M, Ostrikov K. Plasmas meet nanoparticles-where synergies can advance the frotier of medicine. J Phys D: Appl Phys 2011:44:174018.

14. Pawan KT, Kang SK, Kim GJ, et al. Modeling of nanoparticle-mediated electric field enhancement inside biological cells exposed to AC electric fields. Jpn J Appl Phys 2009; 48: 87001.

15. Kim GC, Kim GJ, Park SR, et al. Air plasma coupled with antibody-conjugated nanoparticles: a new weapon against cancer. J Phys D: Appl Phys 2009;42:032005.

16. Hess AR, Hendrix MJ. Focal adhesion kinase signaling and the aggressive melanoma phenotype. Cell Cycle 2006;5:478-80.

17. Sieg DJ, Hauck CR, Ilic D, et al. FAK integrates growth-factor and integrin signals to promote cell migration. Nat Cell Biol 2000;2:249-56.

18. Ozkal S, Paterson JC, Tedoldi S, et al. Focal adhesion kinase (FAK) expression in normal and neoplastic lymphoid tissues. Pathol Res Pract 2009;205:781-8.

19. McLean GW, Carragher NO, Avizienyte $\mathrm{E}$, et al. The role of focal-adhesion kinase in cancer - a new therapeutic opportunity. Nat Rev Cancer 2005;5:505-15.

20. Hess AR, Postovit LM, Margaryan NV, et al. Focal adhesion kinase pro-motes the aggressive melanoma phenotype. Cancer Res 2005;65:9851-60.

21. Short SM, Yoder BJ, Tarr SM, et al. The expression of the cytoskeletal focal adhesion protein paxillin in breast cancer correlates with HER2 overexpression and may help predict response to chemotherapy: a retrospective immunohistochemical study. Breast J 2007;13:130-9.

22. Schmitz KJ, Grabellus F, Callies R, et al. High expression of focal adhesion kinase (p125FAK) in node-negative breast cancer is related to overexpression of HER-2/neu and activated Akt kinase but does not predict outcome. Breast Cancer Res 2005;7:194-203.

23. Wolf-Yadlin A, Kumar N, Zhang Y, et al. Effects of HER2 overexpression on cell signaling networks governing proliferation and migration. Mol Syst Biol. 2006;2:54.

24. Xu X, Liu Y, Wang L, et al. Gambogic acid induces apoptosis by regulating the expression of Bax and Bcl-2 and enhancing caspase- 3 activity in human malignant melanoma A375 cells. Int J Dermatol 2009;48:186-92.

25. Eberle J, Hossini AM. Expression and function of bcl-2 proteins in melanoma. Curr Genomics 2008;9:409-19. 